\title{
Expression of Monomeric C-Reactive Protein in Infarcted Brain Tissue from Patients with Alzheimer's Disease
}

\author{
Mark SLEVIN', Donghui LIU', Glenn FERRIS' ${ }^{1}$, Malik AL-HSINAWI², Raid AL-BARADIE², Jerzy KRUPINSKI \\ 'Manchester Metropolitan University, School of Healthcare Science, MANCHESTER, UNITED KINGDOM, ²Department of Medical Laboratories, \\ Majmaah University, AL MAJMA'AH, SAUDI ARABIA, ${ }^{3}$ Department of Neurology, Hospital Universitari Mutua de Terrassa, BARCELONA, SPAIN
}

\begin{abstract}
Objective: We have previously shown that monomeric-C-reactive protein is deposited in significant quantities within the brain parenchyma after stroke. Since we have recently identified a possible role of this protein in supporting neurodegeneration and aberrant vascular development, we identified a small group of post-mortem brain samples from individuals who had Alzheimer's disease and evidence of tissue infarction/ micro-infarction on histological examination.
\end{abstract}

Material and Method: We used immunohistochemistry staining to identify the monomeric-C-reactive protein expressed in the infarcted brain tissues.

Results: We showed that monomeric-C-reactive protein deposition was highest in those regions affected by stroke or vascular disruption, and that within those same areas, there was more interaction and co-localization between major classical proteins of neurodegeneration ( $\beta$-amyloid and tau).

Conclusion: We hypothesise that vascular disruption and concomitant release of monomeric-C-reactive protein within the brain tissue could exacerbate ongoing neurological damage via stimulation of neuro-inflammation and from direct consequences of its action on both neuronal and vascular cells.

Key Words: Monomeric C-reactive protein, Stroke, Alzheimer's disease, Neurodegeneration, Vascular dementia

\section{INTRODUCTION}

We have previously shown that monomeric C-reactive protein (mCRP) was dramatically over-expressed in the brain extracellular matrix (ECM) of patients following acute ischaemic stroke (1). In addition, mCRP, unlike the native pentameric molecule ( $\mathrm{pCRP}$ ), stimulated aberrant angiogenesis in vitro, induced phosphorylation of tau by neurons and tau-244-372 aggregation in vitro and following injection into the hippocampus of mice, directly resulted in cognitive and memory decline similar to that seen in a model of Alzheimer's disease (AD) $(\operatorname{Tg} \times 3)(2)$.

mCRP also co-localised with CD105 in microvessels suggesting angiogenesis. Phospho-arrays/Western blotting identified signalling activation in both endothelial cells and neurons through $\mathrm{p}$-IRS-1, p-Tau and p-ERK1/2-which was blocked following pre-incubation with mCRP-antibody suggesting that the antibody could have therapeutic potential. mCRP increased vascular monolayer permeability and gap junctions, increased NCAM expression and produced haemorrhagic angiogenesis in mouse matrigel implants and clearly has abnormal effects on the vascular system.

(Turk Patoloji Derg 2017, 33:25-29)

Received : 14.07.2016 Accepted : 05.09.2016
Previously, Strang et al. (3) demonstrated that $A \beta$ plaques generated in vitro were able to dissociate pCRP to mCRP, probably produced de-novo within the brain ECM, whilst in vivo, more mCRP was identified within frontal cortex regions in victims of $\mathrm{AD}$ than normal control brains suggesting a possible pathological or regulatory role in development of the disease.

Cerebrovascular disease, neurovascular dysfunction and cerebral blood flow abnormalities are now recognised as critical influences in the pathophysiological development of $\mathrm{AD}$, damaged, blocked or in patent vessels having a severe effect on the function of local neurovascular units (with approximately $80 \%$ of $\mathrm{AD}$ brains at post-mortem shown to have significant vascular abnormalities).

Damage to the deep penetrating vessels within the brain such as following lacunar stroke, ischaemic stroke or head trauma, may lower the threshold for development of vascular and other (mixed) dementia, particularly in younger patients $(4,5)$.

Correspondence: Mark SLEVIN

Manchester Metropolitan University, School of Healthcare Science,

MANCHESTER, UNITED KINGDOM

E-mail: m.a.slevin@mmu.ac.uk Phone: +44 (0) 1612471172 
Here, we investigated the possible link between mCRP deposition and localization within the brain and indicators of previous stroke or vascular disruption and dementia.

\section{MATERIAL and METHODS}

Samples of brain tissue were obtained from the Bristol Brain Bank, where from a cohort of 10 individuals, 3 were identified as having concomitant histological evidence of $\mathrm{AD}$ and stroke (assessed independently and also by our clinical neurologist; Table I), and a clinical registered history of $\mathrm{AD}$. The $\mathrm{AD}$ cases all had a history of progressive dementia and were selected on the basis of a diagnosis according to Consortium to establish a registry for Alzheimer's disease (CERAD) of 'definite AD' (6). AD neuropathological change was considered a sufficient explanation for the dementia in all cases.

Full ethical approval was obtained for the use of tissue samples as detailed by the South West Dementia Brain Bank (SWDBB) and obtained from them.

Mouse monoclonal antibody to human mCRP sub-unit (M8C10) was obtained from Dr L.A, and was stated as previously (2). Mouse monoclonal antibodies to $\mathrm{p}$-Tau and $\beta$-amyloid were purchased from Abcam (UK). Standard Immunohistochemistry staining was carried out according to the protocols and fully characterized as described previously (1). Paraffin processed sections from frontal, parietal and temporal regions were examined for expression of mCRP (mouse monoclonal; 1:10 dilution; blue-grey colour). These were our own characterized antibodies, and originally a gift from Dr Larry Potempa, Roosevelt University, USA (1). In addition co-staining (double immunohistochemical labelling for key marker proteins of neurodegeneration (rabbit polyclonal p-Tau and $\beta$-amyloid; used at 1:500 dilution; brown) with VIP VECTASTAIN-ELITE-vector HRP kits (from Vector labs. com) was carried out. Particular attention was given to analysis of staining patterns associated with areas of tissue showing evidence of inflammation, vascular damage and regions showing morphological appearance of microinfarct or other previous stroke.
Staining patterns and intensity were observed by an independent neuropathologist in a blinded fashion. Total staining was not quantified but overall expression in different regions and by specific recognisable cell types over the whole region were characterised using our double size sections. For plaque numbers and numbers of neurons positively stained, counting of $10 \times$ fields of view at $\times 100$ per section of three sections was employed. A distinction between strong (intense) and weak staining was also made.

\section{RESULTS}

\section{Immunohistochemical Findings}

The staining pattern revealed that $\mathrm{mCRP}$ was almost absent from normal looking regions of brain tissue without signs of neurodegeneration or previous tissue insult. Occasional blood vessels, neurons and glia of brain tissue showed positive mCRP staining. However, mCRP expression increased in the areas demonstrating typical AD pathology (i.e. amyloid deposits and neurofibrillary tangles). In these later areas, mCRP was mainly localized to blood vessels and neurons with a smaller expression within glia.

mCRP expression was most abundantly present in areas of microinfarction (and adjacent regions) in both grey and white matter, whilst in relatively normal looking areas, expression was weak or none-existent (Figure 1A). $\mathrm{mCRP}$ expression was not seen in normal looking regions and hence quantification of the extent of staining was not attempted.

B-amyloid staining and co-localization: Areas with evidence of previous microinfarctions concomitantly expressed more $\beta$-amyloid. Some of the amyloid plaques were close to adjacent blood vessels where mCRP expression was abundant (Figure 1B). The co-localization between mCRP and $\beta$-amyloid in blood vessels was also mainly seen within areas or adjacent to small infarcts (Figure 1B); however, it was also observed, sporadically in other areas of pure tau pathology without clear infarction in the area.

$\beta$-amyloid pathology within existing plaques was mainly separate, with mCRP-positive 'plaque'-like material generally being distinct from regular $\mathrm{AD}$ plaques, in

Table I: Patient information

\begin{tabular}{lccccc}
\hline Sample ID & Age & Gender & Braak-NFT & CERAD & Infarction \\
\hline $496 \mathrm{~T}$ & 84 & F & 2.3 & - & + \\
\hline $691 \mathrm{~F}$ & 83 & $\mathrm{M}$ & 3.4 & 4 & + \\
\hline $697 \mathrm{P}$ & 87 & $\mathrm{M}$ & 4.4 & 6 & + \\
\hline
\end{tabular}

CERAD: Consortium to establish a registry for Alzheimer's disease. 

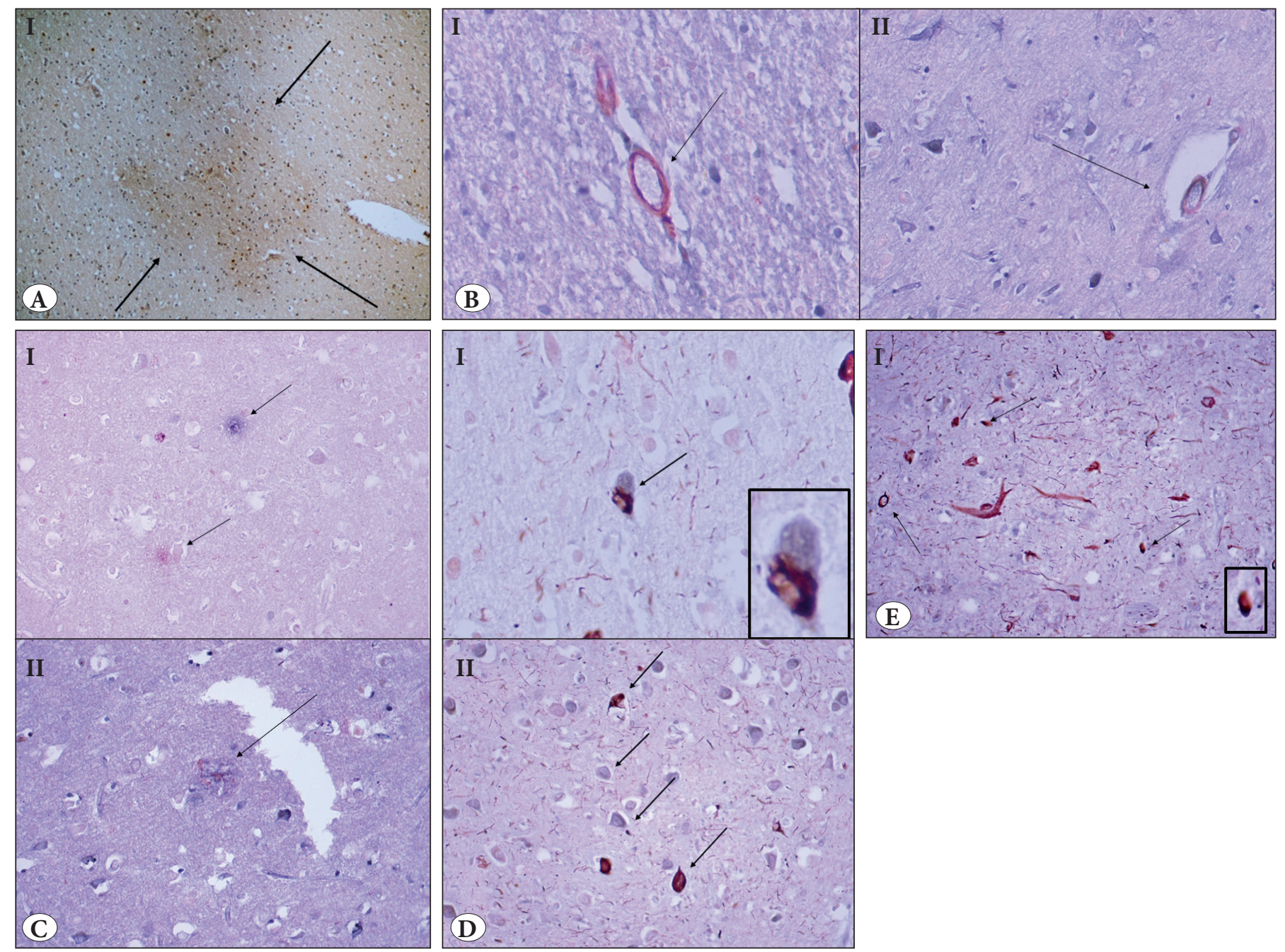

Figure 1: A) Single staining for monomeric C-reactive protein (mCRP)-showing increased expression within a micro-infarcted area (brown, arrows) (mCRP; x100), B) Double labelled sections from previously infarcted regions of white (i) and grey (ii) matter showing co-localization of $\beta$-amyloid (blue-grey) with mCRP (brown) (IHC; 200 ), C) Double staining for $\beta$-amyloid and mCRP showing (i) $\beta$-amyloid-positive (brown) and mCRP-positive (blue-grey) separate plaques in none-infarcted regions (ii) a plaque containing both proteins within a previously infarcted area (arrows) (IHC; x100) (inset x 200) (i) and x 200 (ii)). D) Double staining co-localization within micro-infarcted areas of p-tau (brown) and mCRP (blue-grey) (i) an isolated double-labelled neuron (IHC; x100; inset x 200) and (ii) neurodegenerative grey matter with separately stained neurons for mCRP (blue-grey) and p-Tau (arrows). E) Double staining showing co-localization of amyloid and mCRP (blue-grey)-NFTs-p-Tau (brown) in an infarcted grey matter brain region. (i) areas of colocalization are shown by arrows (IHC; x200).

relatively normal looking tissue regions. However, adjacent to previously infarcted regions some of the plaques (approximately $10 \%$ based on counting of $10 \times$ fields of view at $\times 100$ per section of three sections) contained a combination of the two proteins (Figure 1C).

p-Tau Staining and Co-Localization: The majority of infarcted areas also exhibited other typical AD pathology like tau deposits. Most of the mCRP co-localized primarily with tau within neurons in peri-nuclear regions (Figure $1 D)$, although, again, the majority of neurons were either $\mathrm{p}$-Tau or mCRP positive however the number of neurons co-localising with the two proteins was approximately $(15 \%$ based on counting of $10 \times$ fields of view at $\times 100$ per section of three sections). Co-localisation of the two proteins mCRP and p-Tau was present but generally at different positions within the neurons. (arrows showing dark grey staining) (Figure 1E).

Toxic Tau fibrils (neurofibrillary tangles) were present in some of the most degenerated regions and there was evidence of co-localization of p-tau. Limitations of this 'case study' analysis are clearly from the small numbers of sections and patients reviewed. In addition, the relative 
timing of events in these patients e.g. infarction versus $\mathrm{AD}$ symptoms is not known and so the relative influence of one process on the other is subjective.

\section{DISCUSSION}

Significant expression of mCRP was found adjacent to infarcted areas. This is in line with our previously published observation and hypothesis of a direct link between mCRP deposition and vascular damage in the brain linking it to subsequently and chronically hypo-perfused tissue regions (1). Since mCRP was also found in smaller quantities, in areas that were not directly associated with previous infarction, the expression of mCRP is likely to be due to de novo synthesis similar to that seen by Strang et al (3), however, within blood vessel walls, mCRP could infiltrate directly from the circulation through damaged intimal linings in patients with vascular disease.

$\beta$-amyloid deposition may increase in damaged areas because of chronic inflammation after stroke associated with chronic cerebrovascular dysfunction, and this could be perpetuated at least in part by the presence of excessive mCRP (7-9). Similarly, build-up of $\beta$-amyloid is known to induce neuro-inflammation directly, thus potentially perpetuating the neurodegenerative consequences (6). Since mCRP is known to stimulate aberrant angiogenesis (2), this expression could have a potential negative influence on existing or neo-microvessel function and patency contributing to production of a local hypo-perfused neurodegenerative-friendly environment.

Localization of mCRP with phosphorylated tau in neurons could have physiological significance. Others and ourselves have demonstrated that mCRP can phosphorylate tau (Ser $202,396)$ directly in vitro $(2,10)$, possibly by a mechanism involving GSK3 $\beta$. It is worthy of note that whilst colocalising, the two proteins $\mathrm{mCRP}$ and $\mathrm{p}$-Tau were present generally at different positions within the neurons. However, this still suggests that mCRP could contribute to abnormal activation of these neurons after stroke (2).

In summary, mCRP was not found in normal-looking brain tissue of none dementia patients, however it is produced and laid down in large quantities within the brain following stroke, other brain injury or conditions linked with neuro-inflammation. Given its strong aberrant biological properties associated with neurodegenerative signalling, vascular modulation and angiogenesis, and its direct perpetuation of inflammatory responses, a clear role for this protein in promoting $\mathrm{AD}$ and $\mathrm{VaD}$ is proposed (11).
The findings shown here strengthen this hypothesis further providing case studies of $\mathrm{AD}$ patients where vascular insults probably linked to a local hypoxic environment appear to be highlighted by a strong deposition of mCRP and concomitant disproportionate evidence of localised neurodegenerative disease. Since the majority of patients who suffer serious stroke go on to develop cognitive decline, lower executive function, and psychomotor processing speed; and over $10 \% \mathrm{AD}$ within 5 years $(12,13)$, further research should study potential mechanisms linking the two conditions with a view to creating protective novel therapeutics.

\section{CONFLICT of INTEREST}

The authors declare no conflict of interest.

\section{SOURCE of FUNDING}

This work was supported by the Research Centre of Healthcare Science at Manchester Metropolitan University, and by Sheikh Abdullah bin Abdul Mohsen Al-Tuwaijri project grants within, Majmaah University, Saudi Arabia.

\section{ACKNOWLEDGEMENT}

We would like to thank the South West Dementia Brain Bank (SWDBB) for providing brain tissue for this study. The SWDBB is part of the Brains for Dementia Research programme, jointly funded by Alzheimer's Research UK and Alzheimer's Society and is supported by BRACE (Bristol Research into Alzheimer's and Care of the Elderly) and the Medical Research Council. This research was supported by Sheikh Abdullah bin Abdul Mohsen Al-Tuwaijri project grants within, Majmaah University, Saudi Arabia. The authors would like to express their gratitude towards Sheikh Abdullah Abdul Mohsen Al-Tuwaijri, Rector Dr. Khalid Saad Al Muqrin for providing the necessary support and assistance in completing this piece of work.

\section{REFERENCES}

1. Slevin M, Matou-Nasri S, Turu M, Luque A, Rovira N, Badimon L, Boluda S, Potempa L, Sanfeliu C, de Vera N, Krupinski J. Modified C-reactive protein is expressed by stroke neovessels and is a potent activator of angiogenesis in vitro. Brain Pathol. 2010;20:151-65.

2. Slevin M, Matou S, Zeinolabediny Y, Corpas R, Weston R, Liu D, Boras E, Di Napoli M, Petcu E, Sarroca S Popa-Wagner A, Love, S, Font MA, Potempa LA, Al-baradie R, Sanfeliu C, Revilla S, Badimon L, and Krupinski J. Monomeric C-reactive protein-a key molecule driving development of Alzheimer's disease associated with brain ischaemia? Sci Rep. 2015;5:13281. 
3. Strang F, Scheichl A, Chen YC, Wang X, Htun NM, Bassler N, Eisenhardt SU, Habersberger J, Peter K. Amyloid plaques dissociate pentameric to monomeric $\mathrm{C}$-reactive protein: A novel pathomechanism driving cortical inflammation in Alzheimer's disease? Brain Pathol. 2012;22:337-46.

4. Montagne A, Nation DA, Pa J, Sweeney MD, Toga AW, Zlokovic BV. Brain imaging of neurovascular dysfunction in Alzheimer's disease. Acta Neuropathol. 2016;131:687-707.

5. Toledo JB, Arnold SE, Raible K, Brettschneider J, Xie SX, Grossman M, Kukull WA, Trojanowski JQ. Contribution of cerebrovascular disease in autopsy confirmed neurodegenerative disease cases in the National Alzheimer's coordinating centre. Brain. 2013;136:2697-706.

6. Hyman BT, Phelps CH, Beach TG, Bigo EH, Cairns NJ, Carrillo MC, Dickson DW, Duyckaerts C, Frosch MP, Masliah E, Mirra SS, Nelson PT, Scneider JA, Thal DR, Thies B, Trojanowski JQ, Vinters HV, Montine TJ. National Institute on Aging-Alzheimer's Association guidelines for the neuropathologic assessment of Alzheimer's disease. Alzheimer's Dementia. 2012;8:1-13.

7. McCaulley ME, Grush KA. Alzheimer's Disease: Exploring the role of inflammation and implications for treatment. Int J Alzheimers Dis. 2015;2015:515248.
8. Thiele JR, Habersberger J, Braig D, Schmidt Y, Goerendt K, Maurer V, Bannasch H, Scheichl A, Woollard KJ, von Dobschütz E, Kolodgie F, Virmani R, Stark GB, Peter K, Eisenhardt SU. Dissociation of pentameric to monomeric C-reactive protein localizes and aggravates inflammation: In vivo proof of a powerful proinflammatory mechanism and a new anti-inflammatory strategy. Circulation. 2014;130:35-50.

9. Humpel C. Chronic mild cerebrovascular dysfunction for Alzheimer's disease? Exp Gerentol. 2011;46:225-32.

10. Guo H, Wang H, Wang C, Cheng Y, Zou Z, Li Y, Wu J, Xu J. C-Reactive protein induces Tau Hyperphosphorylation via GSK3 $\beta$ Signalling Pathway in SH-SY5Y Cells. J Mol Neurosci. 2015;56:519-27.

11. Slevin M, Krupinski J. A role for monomeric C-reactive protein in regulation of angiogenesis, endothelial cell inflammation and thrombus formation in cardiovascular disease? Histol Histopathol. 2009;24:1473-8.

12. Desmond DW, Moroney JT,Sano M, Stern Y. Incidence of dementia after ischemic stroke: Results of a longitudinal study. Stroke. 2002;33:2254-62.

13. Mandzia JL, Smith EE, Horton M, Hanly P, Barber PA, Godzwon C, Donaldson E, Asdaghi N, Patel S, Coutts SB. Imaging and baseline predictors of cognitive performance in minor ischemic stroke and patients with transient ischemic attack at 90 Days. Stroke. 2016;47:726-31. 